ARTICLE

DOI: $10.1038 / s 41467-017-01964-9$

\title{
Reading and editing the Pleurodeles waltl genome reveals novel features of tetrapod regeneration
}

\author{
Ahmed Elewa ${ }^{1}$, Heng Wang ${ }^{2}$, Carlos Talavera-López ${ }^{1,7}$, Alberto Joven ${ }^{1}$, Gonçalo Brito (1) ${ }^{1}$, Anoop Kumar ${ }^{1}$, \\ L. Shahul Hameed ${ }^{1}$, May Penrad-Mobayed ${ }^{3}$, Zeyu Yao', Neda Zamani ${ }^{4}$, Yamen Abbas ${ }^{5}$, Ilgar Abdullayev ${ }^{1,6}$, \\ Rickard Sandberg (10 ${ }^{1,6}$, Manfred Grabherr ${ }^{4}$, Björn Andersson (1D ${ }^{1}$ \& András Simon ${ }^{1}$
}

\begin{abstract}
Salamanders exhibit an extraordinary ability among vertebrates to regenerate complex body parts. However, scarce genomic resources have limited our understanding of regeneration in adult salamanders. Here, we present the $\sim 20 \mathrm{~Gb}$ genome and transcriptome of the Iberian ribbed newt Pleurodeles waltl, a tractable species suitable for laboratory research. We find that embryonic stem cell-specific miRNAs mir-93b and mir-427/430/302, as well as Harbinger DNA transposons carrying the Myb-like proto-oncogene have expanded dramatically in the Pleurodeles walt genome and are co-expressed during limb regeneration. Moreover, we find that a family of salamander methyltransferases is expressed specifically in adult appendages. Using CRISPR/Cas9 technology to perturb transcription factors, we demonstrate that, unlike the axolotl, Pax3 is present and necessary for development and that contrary to mammals, muscle regeneration is normal without functional Pax7 gene. Our data provide a foundation for comparative genomic studies that generate models for the uneven distribution of regenerative capacities among vertebrates.
\end{abstract}

\footnotetext{
${ }^{1}$ Department of Cell and Molecular Biology, Karolinska Institute, Stockholm, SE-171 65, Sweden. ${ }^{2}$ College of Animal Science and Technology, Huazhong Agricultural University, Wuhan, 430070, China. ${ }^{3}$ Institut Jacques Monod, CNRS \& University Paris-Diderot, Paris, 75205, France. ${ }^{4}$ Department of Medical Biochemistry and Microbiology, Uppsala University, Uppsala, SE-751 23, Sweden. ${ }^{5}$ Department of Stem Cell and Regenerative Biology, Harvard Stem Cell Institute, Harvard University, Cambridge, MA 02138, USA. ${ }^{6}$ Ludwig Institute for Cancer Research, Stockholm, SE-171 65, Sweden. ${ }^{7}$ Present address: The Francis Crick Institute, NW1 1AT London, UK. Ahmed Elewa, Heng Wang and Carlos Talavera-López contributed equally to this work. Correspondence and requests for materials should be addressed to A.E. (email: ahmed.elewa@ki.se) or to A.S. (email: andras.simon@ki.se)
} 
T he random manifestation of extensive regeneration capacities in the animal kingdom implies a phylogenetically widespread regeneration potential, which is masked in most species $^{1-5}$. Among tetrapods, salamanders, such as newts and axolotls, display the largest regenerative repertoire. A newt can rebuild entire limbs, tails, jaws, cardiac muscle, ocular tissues, and restore central nervous system tissues including brain structures ${ }^{6}$ (Fig. 1). However, it is important to note that major differences exist even among salamanders. In contrast to the paedomorphic axolotl, newts undergo metamorphosis, have a broader regeneration spectrum and mobilize additional cell sources for regeneration of the same body part $^{7}$. Such interspecies differences among closely related species offer opportunities to reveal valuable information about the evolution of processes that allow or counteract regeneration. Although significant progress has been made to characterize salamander transcriptomes and proteomes ${ }^{8-12}$, features such as species-specific genes, expansion or contraction of gene families, and the underlying cause of their gigantic genome size remain largely unexplored. In addition, due to their complex and long life cycle, most newt species are cumbersome to breed under laboratory conditions, which has hampered the establishment of genetically modified lines. However, the Iberian ribbed newt Pleurodeles waltl ( $P$. waltl) is easily bred in laboratories and retains the widest known spectrum of regeneration abilities among adult vertebrates ${ }^{13}$ (Fig. 1; Supplementary Fig. 1a). Here we describe the genome and transcriptome of $P$. waltl (Methods; Supplementary Methods, Supplementary Fig. 1b, and Supplementary Tables 1-5) as a resource to explore regeneration relevant novelties, and adapt CRISPR/Cas9 technology to perturb key transcription factors involved in regeneration.

\section{Results}

Sequencing the genome and transcriptome of $\boldsymbol{P}$. waltl. The diploid genome of $P$. waltl is organized in 12 chromosome pairs, which have been the subject of classical lampbrush chromosome studies $^{14}$ (Supplementary Fig. 1c-h). The $P$. waltl haplotype genome size is $\sim 20 \mathrm{~Gb}$ (Supplementary Table 1 ), making this one of the largest vertebrate genomes sequenced to date. Our genome annotation pipeline identified 14,805 complete protein-coding gene models and we estimate that this set represents $64.8 \%$ of P. waltl protein-coding genes (Methods; Supplementary Methods, Supplementary Fig. 2a). The remaining gene content was reconstructed in the de novo transcriptome assembly involving RNAseq data from embryonic, larval, different adult tissues, and limb regeneration stages (Methods; Supplementary Methods, Supplementary Tables 3-5). We estimate that this combined set of gene models and transcripts represents $98.1 \%$ of the $P$. walt proteincoding repertoire (Supplementary Methods; Supplementary Table 6). To provide a platform for comparative genomic studies, we identified 19,903 orthology groups involving $P$. walt proteincoding genes and/or transcripts (Supplementary Methods; Supplementary Fig. 2a, Supplementary Table 1, Supplementary Data 1). Of these orthology groups, 1575 consisted of salamander members only (salamander groups) and 1130 consisted of salamander and Xenopus orthologs only (amphibian groups). The remaining 17,198 groups consisted of salamander and other vertebrate orthologs (human, mouse, chicken, lizard, or zebrafish). Importantly, we did not observe any expansion or loss of non-transposable protein-coding orthologs compared to other vertebrates (more than twofold increase or decrease) (Supplementary Methods, Supplementary Data 1-3).

An expansion of Harbinger elements in the $P$. walt genome. A striking feature of the $P$. walt genome is the extent and diversity of its repetitive elements. The genome is host to a diverse population of class I and class II transposable elements (Supplementary Table 1). We assembled a repeat library by majority vote $\mathrm{k}$-mer extension ${ }^{15}$, followed by alignment to the repeat database RepBase $^{16}$ using Satsuma ${ }^{17}$, yielding 428 distinct sequences (Methods). Gypsy retrotransposons are the most
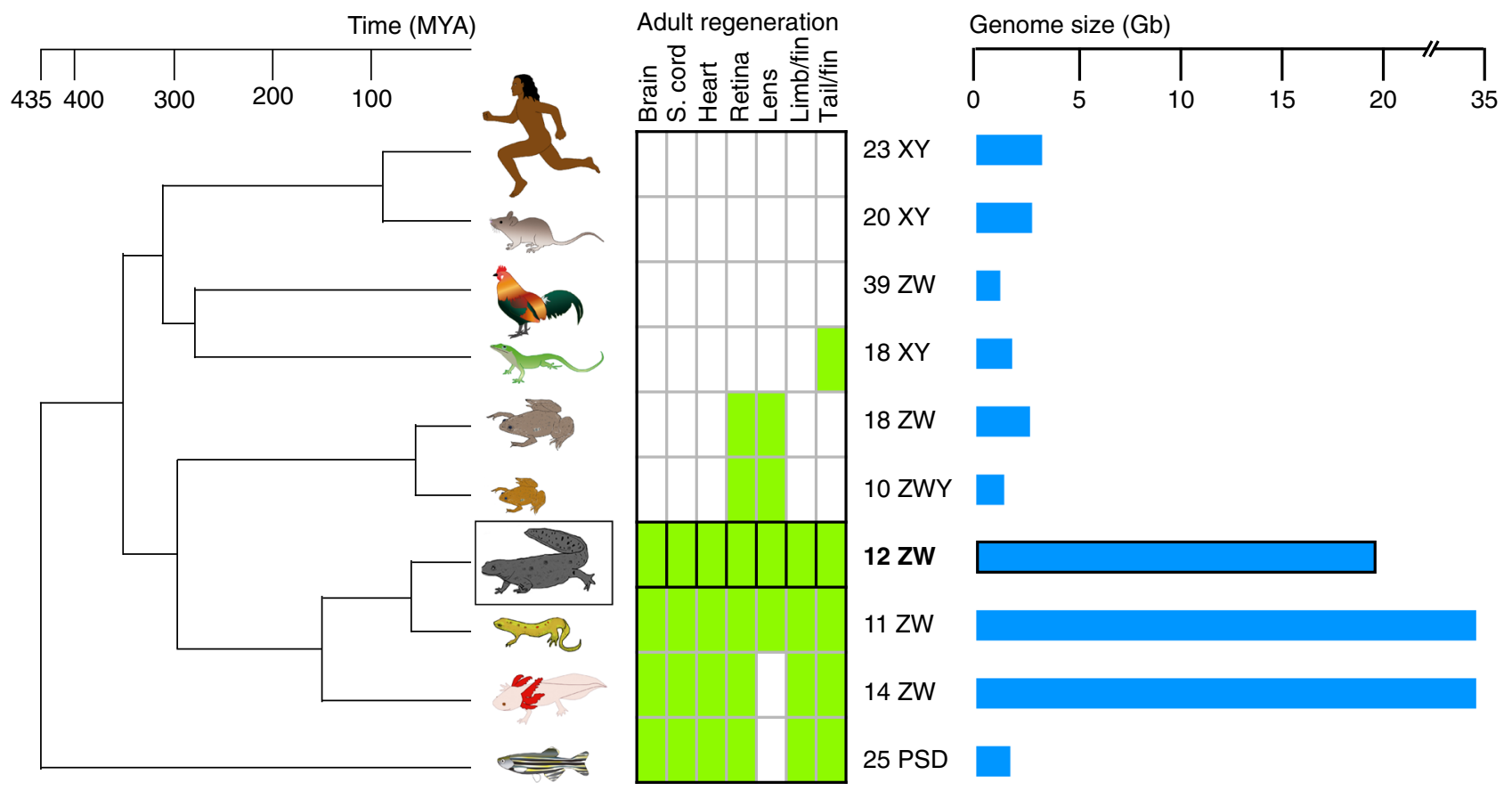

Fig. 1 The Iberian ribbed newt $P$. walt/ is a prime model for adult regeneration. Compared to other research animal models, $P$. walt/ is the most regenerative adult vertebrate amenable to laboratory breeding. Phylogenetic tree from TimeTree ${ }^{66}$. Regeneration capacities based on ${ }^{6,13,67-70}$. Sex chromosomes from refs. ${ }^{71-74}$. Genome sizes from https://www.ncbi.nlm.nih.gov/genome/ (summed from Size (Mb) column) 
frequent repetitive elements in $P$. waltl, followed by the Harbinger transposons, and together account for about two thirds of the genome repetitive content (Supplementary Table 1). A phylogeny of 1200 Gypsy elements longer than one kilobase indicates continuous expansion of this family (Fig. 2a, b), while Harbinger elements have undergone two distinct evolutionary bursts, with one recent expansion, visible from the distribution of pairwise similarity (Fig. 2a, b; Supplementary Fig. 2b). Harbinger elements are distinct from other transposons in that they carry a $M y b$-like gene, a proto-oncogene that acts as a transcription factor. While Harbinger elements gave rise to the genes Harbi ${ }^{18}$ and Naif $1^{19}$ in the vertebrate lineage, their contribution to vertebrate genome content is extremely rare with the leading example being the genome of coelacanth Latimeria chalumnae ( $\sim 1$ to $4 \%$ of the genome $)^{20}$. Therefore, the Harbinger element expansion we describe in $P$. waltl is hitherto unprecedented. a Gypsy
6000 sequences $>1 \mathrm{~kb}$

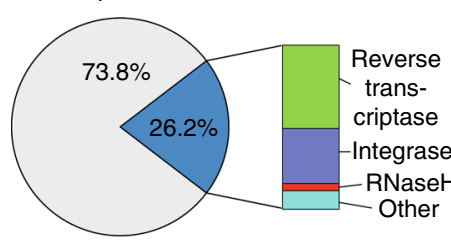

b
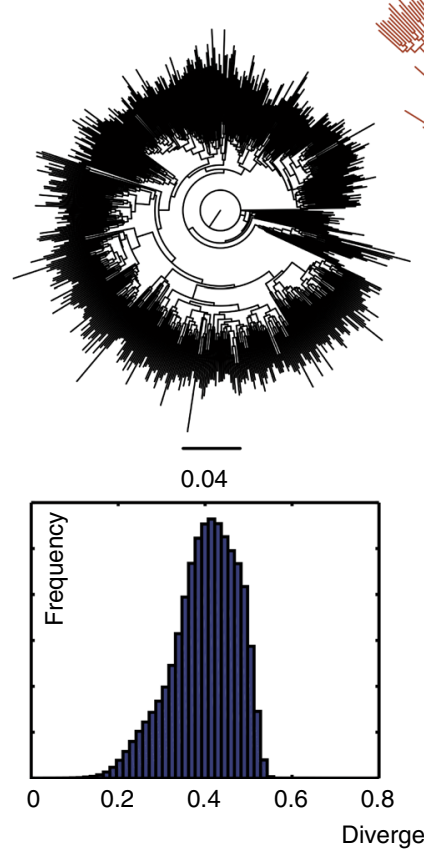

Divergence
Harbinger

1805 sequences $>1 \mathrm{~kb}$

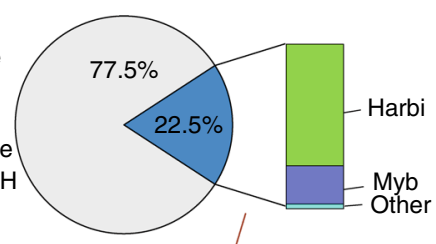

C
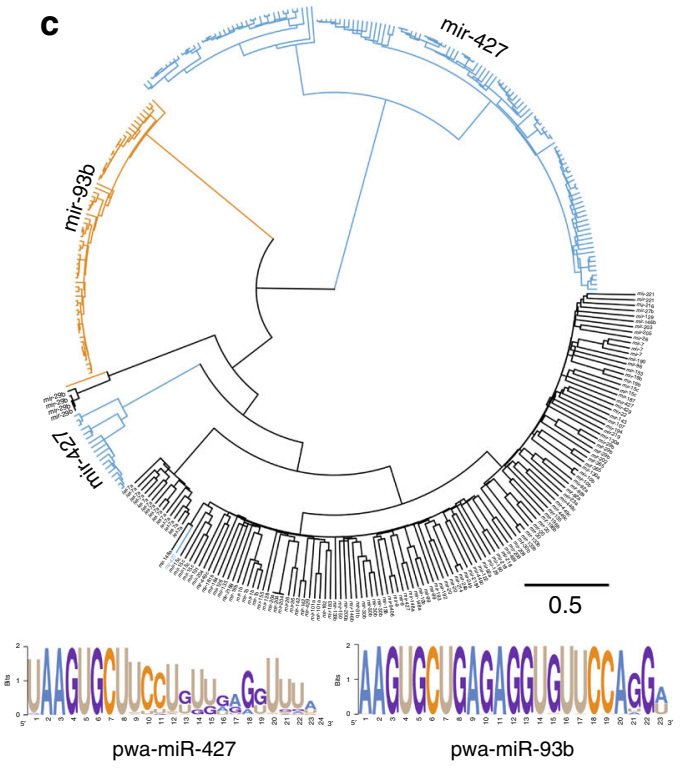

d

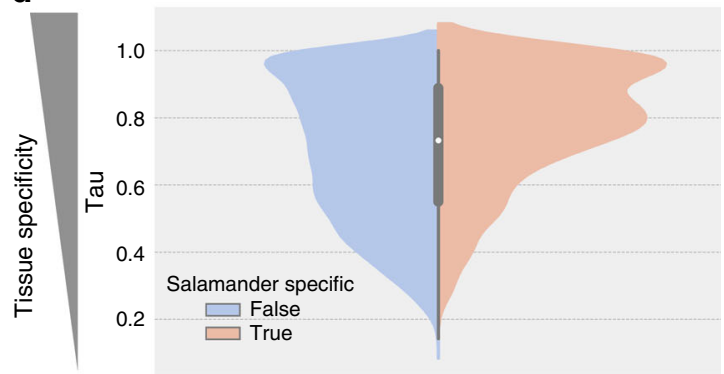

e

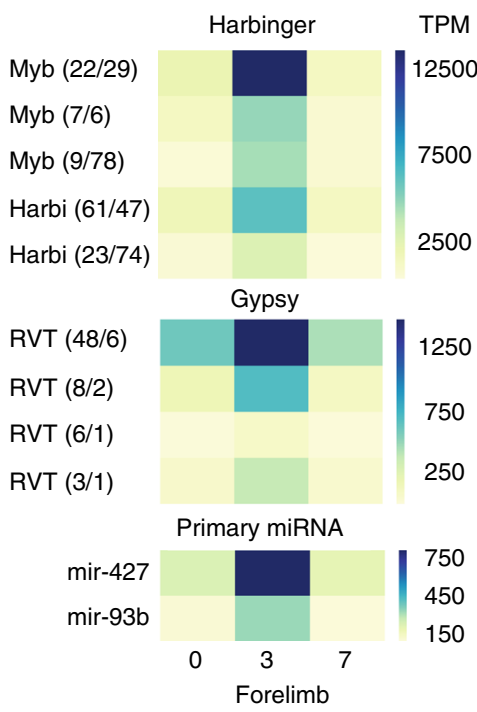

f

Mtase (1/1)

Mtase (2/2)

Mtase (1/1)

Mtase (5/2)

Mtase (5/2)

Mtase (2/1)

Mtase (3/1)

Mtase (5/1)

Mtase (1/2)

Mtase (1/1)

Mtase (2/1)

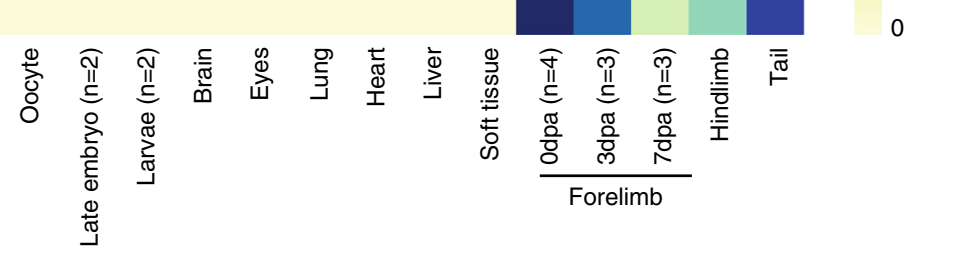

NNMT/PNMT/TEMT Methyl-transferase signature

TPM

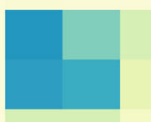

320

240

160
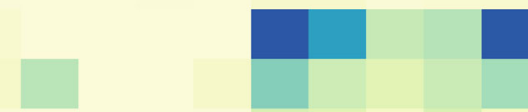

80

(days post amputation) 
Expansion of embryonic stem cell-specific miRNAs in P. waltl. MicroRNAs (miRNAs) are short non-coding RNAs that regulate post-transcriptional gene expression ${ }^{21-23}$. A number of miRNAs, including miR-302, are capable of reprogramming mammalian somatic cells into an induced pluripotent state ${ }^{24}$. We identified 361 miRNA precursors in the $P$. walt genome that produce 67 distinct mature miRNAs conserved in Xenopus. In addition, the de novo transcriptome included 202 transcripts bearing conserved miRNA precursors (primary miRNA transcripts) that produce 55 of the 67 genome predicted mature miRNAs (Supplementary Methods, Supplementary Fig. 3, Supplementary Tables 7-8, Supplementary Data 4). Surprisingly, most of the genome predicted precursors were copies of mir- $93 \mathrm{~b}^{25}$ and mir$427^{26}$ (known as mir-430 in zebrafish ${ }^{27}$ and mir-302 in mammals $^{28}$ ) (66 and 155 precursors, respectively) (Fig. 2c). Both mir$93 \mathrm{~b}$ and mir-427 bear the characteristic embryonic stem cellspecific cell cycle regulating (ESCC) seed (AAGUGC) and are of interest to regeneration studies ${ }^{29}$ (Fig. 2c). The expansion of mir427 has been reported in Xenopus ( $\sim 80$ copies) ${ }^{25}$ and zebrafish $(\sim 100 \text { copies })^{30}$ and thus predates the Devonian period ${ }^{31}$. The mir-93b expansion, however, appears to be salamander-specific and we place its occurrence in the Jurassic period or later ${ }^{32}$.

Harbinger and ESCC miRNA expression during limb regeneration. Given the compelling expansion of ESCC miRNAs and Harbinger transposable elements, we analyzed whether mir-93b, mir-427, Myb, and Harbi domain containing genes are regulated during adult $P$. waltl limb regeneration (Supplementary Table 9). We mapped total RNAseq reads from three early developmental stages, nine adult ( $>2$ years old) body parts and two limb regeneration stages; 3 and 7 days post amputation (Supplementary Table 3) to 14,805 gene models and 108,713 transcripts belonging to the $P$. waltl orthology groups in addition to 202 miRNA primary transcripts (Supplementary Methods, Supplementary Data 1,4$)$. We found that mir-93b and mir-427 primary transcripts are upregulated in the regenerating limb 3 days post amputation $(p<0.001)$ and that their mature miRNAs are detected during limb regeneration (21 and 45 reads per million, respectively) (Fig. 2e; Supplementary Fig. 4, Supplementary Table 9 and Supplementary Data 4). In addition, we found that five orthology groups containing Myb or Harbi domains were upregulated at 3 days post amputation $(p<0.001)$ (Supplementary Table 9) similar to mir-93b and mir-427 primary transcripts (Fig. 2e). On the contrary, the two vertebrate Harbinger derivative genes Naif1 and Harbil were expressed at low levels in all our datasets and showed no regulation during regeneration (Supplementary Table 10). Four Gypsy orthology groups were also upregulated 3 days post amputation $(p<0.01)$, however, their expression levels were an order of magnitude lower than the Harbingers (Supplementary Table 9). Transposable elements are often domesticated to benefit their hosts and RNA transposable element expression has been described in mammalian pluripotent stem cells $s^{33}, 34$. Our data illustrate that the both RNA and DNA transposons, Gypsy and Harbinger, respectively, in addition to ESCC miRNAs respond to adult salamander injury. The extent to which they co-regulate regeneration awaits further studies.

Restricted expression of salamander methyltransferases. Genes found only in salamanders may offer insight into their unique regenerative abilities. Our annotation pipeline identified 1545 orthology groups that consisted of genes and transcripts in the $P$. waltl genome and at least one other salamander, but no sequences from the other seven model vertebrates analyzed. We consider these orthology groups to be putatively salamander-specific and refer to them by the protein domain(s) detected in their members. We calculated the tau score ${ }^{35}$ (an indicator of tissue specificity) for all orthology groups and found that groups with only salamander genes are more tissue specific than groups with orthologs in other vertebrates (Fig. 2d and Methods). Surprisingly, we found eleven salamander orthology groups encoding a NNMT/ PNMT/TEMT methyltransferase domain and expressed specifically in adult limbs and tail, four of which were significantly downregulated on the seventh day of regeneration $(p<0.001$; Fig. 2f; Supplementary Fig. 2b and Supplementary Table 9). No other salamander orthology groups showed this expression pattern. Together, our data show that $P$. waltl has evolved genes with tissue-restricted expression profiles and that target methylation may have evolved in a manner consistent with limb regeneration.

$P a x 3$ and $P a x 7$ are present and functional in $P$. waltl. We were intrigued by the absence of Pax3 in the axolotl (Ambystoma mexicanum) transcriptome, genome and previous gene expression studies ${ }^{10,36-38}$. Pax3 is a paired-end homeodomain transcription factor required for early development ${ }^{39,40}$ and its paralog Pax7 regulates skeletal muscle regeneration ${ }^{7}, 41$. Manual curation of the Pax gene family and in situ hybridization confirmed the presence of Pax3 and Pax7 in P. waltl (Supplementary Fig. 5a-c). We adapted CRISPR/Cas9 technology to P. walt and mutated both genes (Methods; Supplementary Fig. 5a,d and Supplementary Table 11). Whereas PaxT $7^{-1}$ compound heterozygote $F_{1}$ animals exhibited no apparent deficiencies in muscle development, Pax3 mosaic-mutants died or developed animals with several developmental anomalies, exemplified by muscle-less limbs (Fig. 3a, b; Supplementary Fig. 5e-f and Supplementary Video 1). Next we asked how these mutations affect limb regeneration. Pax $T^{-1-} \mathrm{F}_{1}$ animals regenerated limbs indistinguishable from wildtype controls, including skeletal muscle and cells in satellite cell position (Fig. 3c, d and Supplementary Fig. 6; Methods). Pax3 mosaic $\mathrm{F}_{0}$ mutants lacking limb skeletal

\footnotetext{
Fig. 2 Genomic expansion and the expression of Harbinger DNA transposable elements pluripotency miRNAs and salamander-specific methyltransferases during adult limb regeneration in $P$. waltl. a Portion of Gypsy and Harbinger repeats ( $>1 \mathrm{~kb}$ ) retaining a detectable protein domain. $\mathbf{b}$ Phylogeny of Gypsy and Harbinger elements. While the tree-based on Gypsy elements indicates a uniform expansion, the tree-based on Harbinger elements shows three branches (brown) resulting from a second distinct burst. The second Harbinger burst also results in a bimodal pairwise genomic distance distribution, compared to a unimodal distribution for the Gypsy elements. c Phylogenic tree of 361 mature $P$. walt/ miRNAs with miR-427 and miR-93b expansion highlighted (top). Sequence logo based on multiple alignment of 155 miR-427 sequences and 66 miR-93b sequences (bottom). Note the embryonic stem cell-specific cell cycle regulating seed AAGUGC within the first eight nucleotides. $\mathbf{d}$ Split violin plot showing the tau score distribution of $P$. waltl genes that have non-salamander vertebrate orthologs (blue) and genes that only have salamander orthologs (beige). e RNAseq quantification of five Harbinger orthology groups encoding Myb or Harbi domain-bearing proteins (top), four Gypsy orthology groups encoding reverse transcriptase domain-bearing proteins (middle) and mir-427 and mir-93b primary transcripts (bottom). In parentheses are the number of transcripts and gene models belonging to each group. The groups chosen were significantly upregulated in the regenerating limb 3 days post amputation (dpa). Differential expression calculated by DESeq2 (Supplementary Table 9). TPM transcripts per million. $\mathbf{f}$ Eleven orthology groups of salamander methyltransferase are expressed specifically in appendages of the adult animal. In parentheses are the number of transcripts and gene models belonging to each group
} 
a
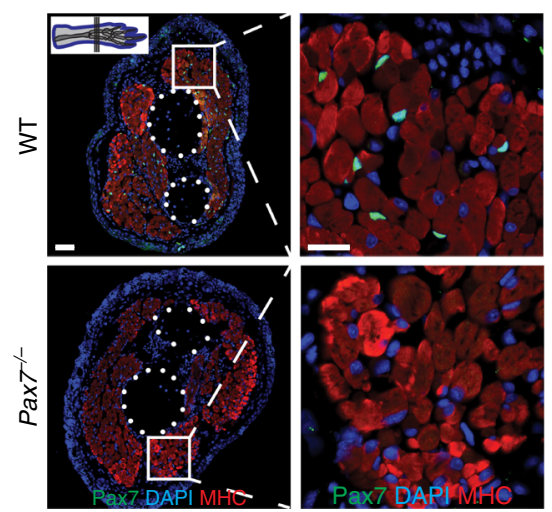
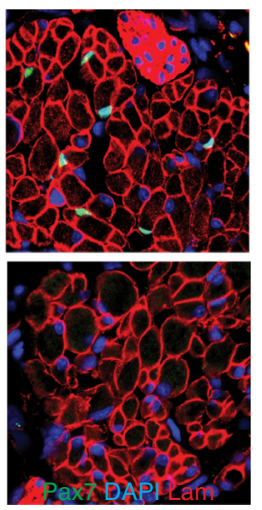

b
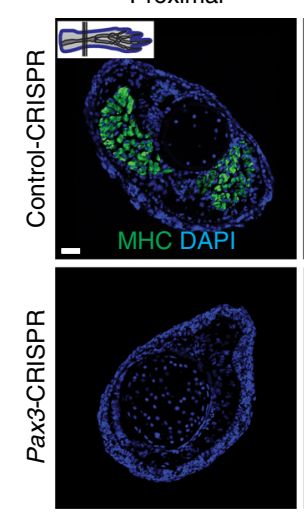
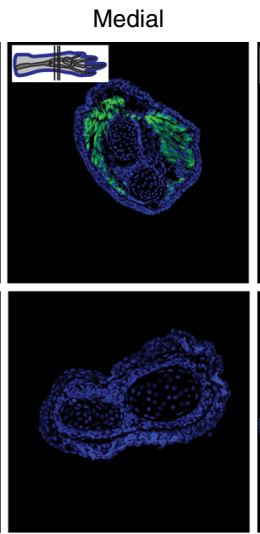
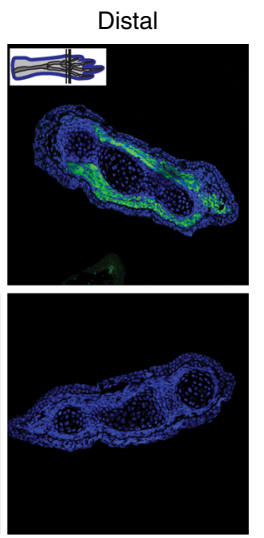
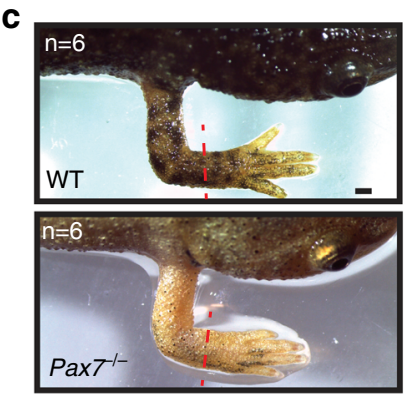

d

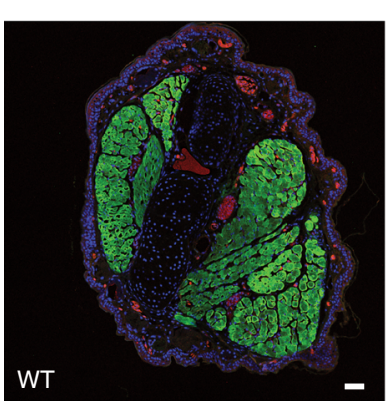

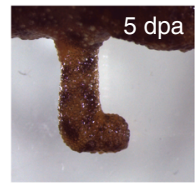
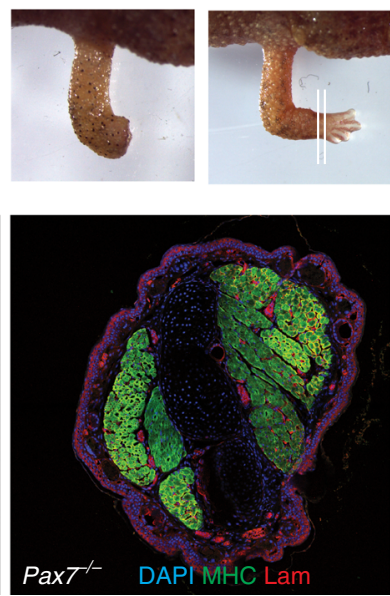
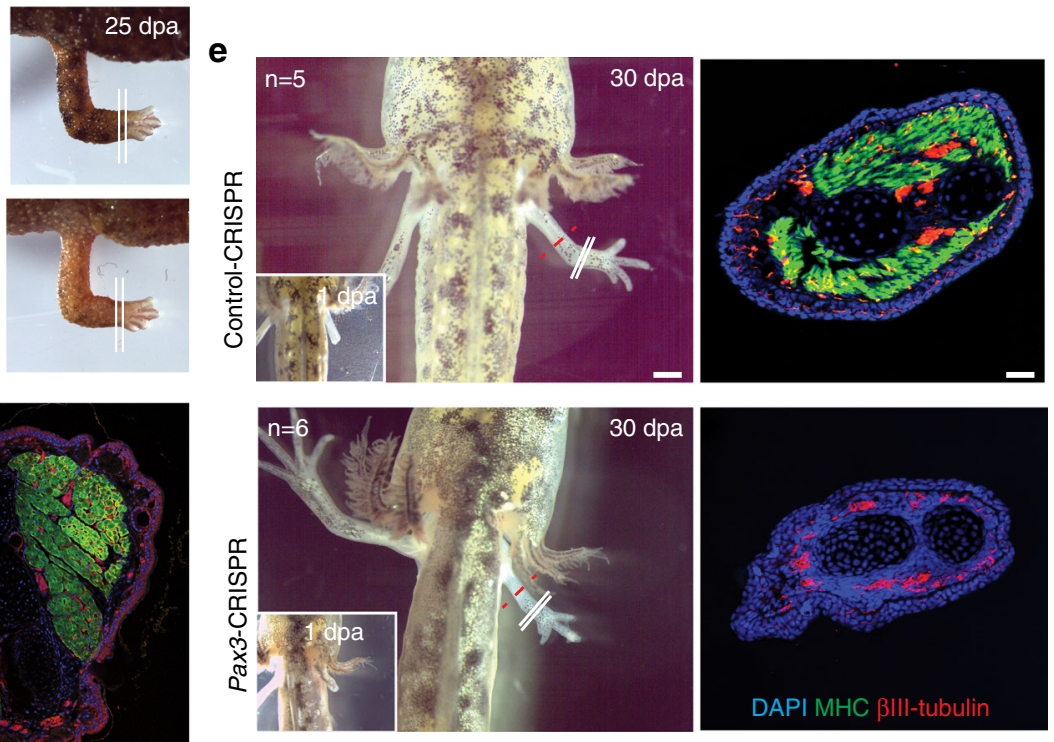

Fig. 3 Newt limb muscle development and regeneration is independent of Pax7. a Pax $7^{-/-} F_{1} P$. walt have normal limb skeletal muscle. Note the similar myosin heavy chain (MHC) and laminin (Lam) staining in Pax ${ }^{-1-}$ and WT animals. Scale bars $=200 \mu \mathrm{m}$ in the overview and $20 \mu \mathrm{m}$ in the insert. $\mathbf{b}$ Pax3CRISPR mosaic animals have muscle-less limbs. Immunofluorescent staining for MHC in transverse sections of Pax3-CRISPR and control-CRISPR limbs.

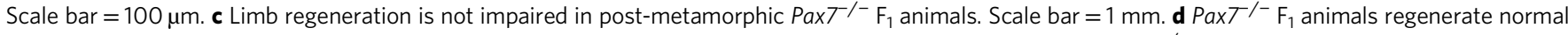
skeletal muscle in the new limb. Note the indistinguishable myosin heavy chain (MHC) and laminin staining in Pax $7^{-/-}$and $\mathrm{WT}$ animals. Scale bar $=100 \mu \mathrm{m}$. e muscle-less limbs in Pax3-CRISPR mosaic animals (stage 45) regenerate muscle-less limbs. Immunofluorescent staining for MHC and the neuronal marker $\beta$ III-tubulin in transverse sections. Scale bar $=1 \mathrm{~mm}$ in the left panels showing animals and $100 \mu \mathrm{m}$ in the right panels showing sections. Red dotted lines indicate amputation plane. White lines indicate section plane

muscle regenerated morphologically normal muscle-less limbs without any other overt phenotypes (Fig. 3e).

\section{Discussion}

Salamander genomes are gigantic, ranging in size between 14 and $120 \mathrm{~Gb}^{42}$. While this feature has facilitated genome structure studies using lampbrush chromosomes ${ }^{43}$, it has delayed sequencing projects and impeded regeneration studies. Lampbrush chromosomes epitomize genome structural plasticity, while salamander regeneration is a prime example of cellular plasticity. Whether these two forms of plasticity are mechanistically related is now a pursuable question.

Reading and editing the $P$. walt genome reveals several new features of limb regeneration and genome evolution. Our loss of function data on $P a x 3$ and $P a x 7$ paralogues show that limb regeneration is not dependent on skeletal muscle and that no other tissues give rise to muscle during limb regeneration in
P. walt $^{44}$. Furthermore, the expression and developmental functions of $P a x 3$ are conserved between $P$. walt and mammals ${ }^{41}$, which also raises the question whether the lack of Pax3 in the axolotl confers additional functions for Pax7. Mammalian skeletal muscle regeneration is impaired in the absence of $P a x 7^{45}$ but we have found that Pax7 loss of function does not impair muscle regeneration in $P$. waltl. In this context, it is important to note that a distinctive process during newt limb regeneration is the reversal of terminal differentiation of skeletal muscle fibers. Myogenic dedifferentiation during limb regeneration produces progenitor cells that build up the new muscle and thus has the potential to compensate for the potential loss of contribution from satellite cells ${ }^{7}$. However cell tracking studies of satellite cells and their progeny are necessary to resolve this question ${ }^{46}$.

The genomic expansion and expression of ESCC miRNAs and Myb domain-bearing Harbingers, along with limb-specific expression of putatively salamander-specific methyltransferases present new possibilities to uncover mechanisms of adult 
vertebrate regeneration using $P$. walt as a model organism. For example, the multitude of mir-427 copies in fish and amphibian genomes in addition to that of mir-93b in newts may provide an opportunity for individual copies to evolve and acquire expression rights and co-regulate a regenerative response after injury. In $X$. laevis and zebrafish, miR-427/430 helps clear of cache of maternally supplied RNA when embryos transition to rely on their own gene expression ${ }^{47,48}$. One intriguing possibility is that miR-427 performs a similar function in $P$. walt during limb regeneration. In this model, miR-427 clears the cytoplasmic cache of mRNAs, thereby erasing a cell's "working memory" and allowing dedifferentiation to occur, while the "deep memory" of the cell's identity and function would not be lost by a permanent inactivation of gene expression.

\section{Methods}

Genomic DNA isolation. High molecular weight genomic DNA was purified from a single larva after discarding the digestive system. The larva represents the first generation of our P.waltl colony, established from fertilized eggs produced in a laboratory colony located in Madrid, Spain. The larva is therefore the fourth/fifth generation of laboratory-bred $P$. waltl, originally obtained with permission from a wild population in Doñana National Park (Spain) for research purposes by Agustin Gonzalez. The body was segmented into five pieces and each piece was placed in $1.5 \mathrm{~mL}$ centrifuge tube. A total of $700 \mu \mathrm{L}$ digestion buffer $(50 \mathrm{mM}$ Tris- $\mathrm{HCl} \mathrm{pH} 8.0$, $100 \mathrm{mM}$ EDTA, $100 \mathrm{mM} \mathrm{NaCl}, 1 \% \mathrm{SDS}$ ) was added to each sample. $50 \mu \mathrm{L} 10 \mathrm{mg}$ $\mathrm{mL}^{-1}$ Proteinase $\mathrm{K}$ was added to the sample and incubated $55-60{ }^{\circ} \mathrm{C}$ overnight with mixing. Another $50 \mu \mathrm{L}$ of $10 \mathrm{mg} \mathrm{mL}^{-1}$ Proteinase $\mathrm{K}$ was added in the morning and the samples incubated for 4 more hours. Next, $20 \mu \mathrm{L} 10 \mathrm{mg} \mathrm{mL}^{-1}$ RNase A (DNase-free) was added to the sample and incubated at $37^{\circ} \mathrm{C}$ for $1-2 \mathrm{~h}$. The solution was transferred to a pre-spun $(1500 \times g$ for $1-2 \mathrm{~min})$ phase lock gel (PLG) $2 \mathrm{~mL}$ Heavy tube. $0.5 \mathrm{~mL}$ phenol-chloroform-isoamyl alcohol (25:24:1) was added to the sample in the PLG $2 \mathrm{~mL}$ tube and mixed well by repeated inversion. Next, tubes were centrifuged at full speed for $5 \mathrm{~min}$ in a microcentrifuge. The resultant aqueous phase was transferred to a fresh pre-spun PLG $2 \mathrm{~mL}$ Heavy tube. A total of $0.5 \mathrm{~mL}$ chloroform-isoamyl alcohol (CI, 24:1) was added to the sample in the PLG $2 \mathrm{~mL}$ tube and mixed well by repeated inversion. Tubes were centrifuged at full speed for $5 \mathrm{~min}$ in a microcentrifuge. The resultant aqueous phase was transferred to a fresh microcentrifuge tube. $100 \%$ isopropanol was added and mixed thoroughly by repeated inversion. High molecular weight DNA was recovered using a heat-sealed glass micropipette tip and transferred to a $1.5 \mathrm{~mL}$ microcentrifuge tube containing $70 \%$ ethanol. The glass tip with DNA was then dipped into $95 \%$ ethanol twice. DNA was allowed to dry partially before being pushed off the tip into a microcentrifuge tube containing $400 \mu \mathrm{L}$ TE and resolubilized overnight. DNA quality was assessed using DropSense and gel electrophoresis.

Karyotyping. Cells were arrested in metaphase using classical procedures, standardized for $P$. walt $t^{49}$ : stage 20 embryos $(n=6)$ were placed in saline solution (30 $\mathrm{mM} \mathrm{NaCl}, 0.34 \mathrm{mM} \mathrm{KCl}, 0.9 \mathrm{mM} \mathrm{CaCl}_{2}$ ) containing $0.5 \%$ colchicine. After $18 \mathrm{~h}$ at $21{ }^{\circ} \mathrm{C}$, the embryos were fixed with a mix of $50 \%$ acetic acid/ $50 \%$ EtOH for $3-5$ min, washed in distilled water and transferred to $50 \%$ acetic acid, were they were dissociated mechanically with a Pasteur glass pipette. Cells were then dropped on Superfrost slides, let dry, and stained with DAPI for confocal analysis. For Supplementary Figure 1, a Z-stack confocal projection was made with an interval of $0.57 \mu \mathrm{m}$ between planes, and the twelve pairs of chromosomes were identified and distributed in three groups according to ref. ${ }^{50}$. For an easy interpretation of the reader, colors were given applying color filters to individually selected chromosomes using Adobe Photoshop. The full extent of the chromosomes in the Zprojection was then selected, filled with color, and organized to provide the karyotype.

Lampbrush chromosome analyses. Ovarian biopsies were performed on adult females of $P$. walt that were anesthetized in 0.15\% MS222 (Amino-benzoic Acid Ethyl, Fluka). Stage IV-VI oocytes were selected and maintained in MBS buffer (Modified Barth's solution) at $18^{\circ} \mathrm{C}$. Germinal vesicles were manually isolated from the oocytes and dissected in $75 \mathrm{mmol} \mathrm{L}^{-1} \mathrm{KCl}, 25 \mathrm{mmol} \mathrm{L}^{-1} \mathrm{NaCl}, 0.01 \mathrm{mmol} \mathrm{L}^{-1}$ $\mathrm{MgCl} 2$ and $0.01 \mathrm{mmol} \mathrm{L}^{-1} \mathrm{CaCl}_{2}, \mathrm{pH} 7.2$, and LBCs were prepared as previously described ${ }^{51}$. Nuclear spread preparations were centrifuged at $300 \times g$ for $10 \mathrm{~min}$, and at $3000 \times g$ for $30 \mathrm{~min}$ at $4{ }^{\circ} \mathrm{C}$. Standard transmitted light were performed at the ImagoSeine core facility (member of the IBiSA and the France-BioImaging (ANR-10-INBS-04) infrastructure) using a wide field Leica microscope with phase contrast plan Apo oil objectives: $\times 40(\mathrm{NA}=1.3), \times 63, \times 100(\mathrm{NA}=1.4)$. Images were captured using a CoolSnap HQ, Photometrics camera driven by the software Metamorph6 (Universal imaging). Series of 11 confocal Z-planes (0.7 $\mu \mathrm{m}$ distance) were collected. Pixel size of the images were $0.1625 \mu \mathrm{m}, 0.103 \mu \mathrm{m}$, and $0.065 \mu \mathrm{m}$ for the $\times 40, \times 63$, and $\times 100$ objectives, respectively.
Genome size calculation. Nuclear content was measured using Vicia faba nucle as reference (2C DNA content $=26.9 \mathrm{pg})^{52}$ (Supplementary Fig. 1). All samples were prepared according to ${ }^{52}$ and stained with 4,6-diamidino-2-phenylindole (DAPI) and measured using a BD Influx ${ }^{\mathrm{TM}}$ by Becton Dickinson flow cytometer. The lasers used for detection and excitation were $488 \mathrm{~nm}$ and $355 \mathrm{~nm}$, respectively. From the mean peak positions of $P$. waltl $(20,255)$ and $V$. faba $(13,746)$ the $2 \mathrm{C}$ DNA content was calculated as $39.6 \mathrm{pg}$ using the equation:

$P$.waltl $2 \mathrm{C}$ value $=V$.faba $2 \mathrm{C}$ value (reference) $) \times((P$.walt $2 \mathrm{C}$ mean peak position $) /($ V.faba $2 \mathrm{C}$ mean peak position))

Based on the equation:

haploid genome size $=(0.978 \times 2 \mathrm{C}$ value $) / 2$, the haploid $P$. walt genome size was calculated to be $19.38 \mathrm{~Gb}$ (Supplementary Fig. 1).

Our estimates agree with Licht and Lowcock $(2 \mathrm{C} \text { value }=42.7 \mathrm{pg})^{53}$ Morescalchi and Olmo $(2 \mathrm{C} \text { value }=39 \mathrm{pg})^{54}$ and are lower than Lizana and collegues $(2 \mathrm{C} \text { value }=48.25 \mathrm{pg})^{55}$

Short read genome sequencing. Libraries for Illumina sequencing were generated and sequenced at SciLifeLabs (Stockholm). Two Illumina TruSeq PCR-free libraries (insert size $180 \mathrm{bp}$ ) were prepared and paired-end $2 \times 125 \mathrm{bp}$ sequencing was done on HiSeq2500 (HiSeq Control Software 2.2.58/RTA 1.18.64) with a setup in High Output mode (Supplementary Table 2).

Genome assembly strategy. Illumina paired-end reads from two $180 \mathrm{bp}$ insert size genomic libraries were assembled into contigs using Abyss (28) using a $k=71$ Illumina paired-end RNAseq reads from representative body sections of $P$. walt were assembled into transcripts using Trans-Abyss $(29)(k=69)$ with the aim to reconstruct only longer, complete transcripts rather than isoforms or short RNAs Trans-Abyss transcripts were then used to scaffold the genomic Abyss-contigs in a third assembly aimed at improving the reconstruction of gene content. The final assembly—denominated $\mathrm{Pw}$ v4.2-was $19.66 \mathrm{~Gb}$ in size and included $\sim 66$ million contigs and 106,895 scaffolds (Table 1). Approximately $45 \%$ of the genome assembly was captured in contigs ranging between $1 \mathrm{~kb}$ and $10 \mathrm{~kb}$ in size and $\sim 6 \%$ $(1.2 \mathrm{~Gb})$ were assembled in contigs and scaffolds longer than $10 \mathrm{~kb}$, the longest being $438.8 \mathrm{~kb}$ in size (Supplementary Fig. 1b).

Repeat library assembly. We assembled a repeat library by majority vote k-mer $(k=25)$ extension algorithm ${ }^{15}$ to the $P$. waltl genome assembly Pw_v4.2, resulting in a set of 428 consensus repeat sequences larger than $1000 \mathrm{nt}$. Out of these, 349 were identified as known repeats through nucleotide alignments against RepBase (version 21.10 downloaded 10/23/2016) ${ }^{16}$ using Satsuma (https://sourceforge.net/ projects/satsuma/ last update $2016-12-14)^{17}$ and selecting the longest alignments respectively if multiple overlapping hits were reported.

Phylogeny of Gypsy and Harbinger elements. We computed pairwise genomic distances using Satsuma for 6000 randomly selected Gypsy and Harbinger elements longer than 1000 nucleotides, requiring alignments to cover the same region in each element. This resulted in 625 Gypsy and 567 Harbinger elements, for which we built phylogeny using the neighbor joining method implemented in phylip ${ }^{56}$, and rooting the tree on midpoint. For bootstrapping, the longest 44 and 50 sequences for Gyspy and Harbinger elements, respectively, were aligned using MUSCLE $^{57}$, from which Maximum Likelihood trees were built using PhyML ${ }^{58,59}$ Bootstrap values were estimates from 100 trials.

Transcriptome RNA preparation and sequencing. 25 indexed Illumina TruSeq Stranded total RNA Zero-Gold libraries (mean insert size $150 \mathrm{bp}$ ) from 20 P. walt body parts and regeneration stages were combined into four pools and sequenced on 4 lanes (one pool per lane) on Illumina HiSeq High Output mode v4, PE $2 \times$ $125 \mathrm{bp}$ at the Science for Life Laboratory, Stockholm (Supplementary Table 3). In all cases, RNA was isolated using Trizol after grinding the sample with a mortar and pestle in liquid nitrogen and precipitated overnight in isopropanol. RNA integrity was assessed using Agilent Bioanalyzer. Samples with RIN $\geq 8.0$ were used for library preparation.

Genome annotation generation of gene models with MAKER. MAKER was used in a two-step approach to identify gene models in the P. waltl genome.

MAKER Tier 1: The clustered assembled transcripts, the Augustus hinted gene models and the protein sequences from uniref 90 were used as input evidence to build the annotation with MAKER.

MAKER Tier 2: Nucleotide gene models from the MAKER Tier 1 annotation were used as input for the second round of gene annotation, instead of the Augustus hinted gene models.

In total, 79,916 gene models were identified using this approach. 19,915 gene models aligned with a transcript in the de novo assembled transcriptome (percent identity $>98 \%$, alignment length $>99 \mathrm{bp}$ ).

Genome editing. Cas9 mRNA and gRNA were synthesized using mMESSAGE T7 ULTRA Transcription Kit (Invitrogen) and MEGAshortscript T7 Transcription Kit (Invitrogen), respectively. Genomic DNA was extracted with Qiagen DNAeasy kit. 
The PCR products were cloned into TOPO cloning vector (Invitrogen). Individual clones were sequenced with T7 primer (Supplementary Table 9).

Single-cell fertilized eggs were obtained by either natural or induced breeding and injected according to previously published protocols with modifications ${ }^{60,61}$. Briefly, 500 pg Cas9 RNA and 100 pg gRNA were mixed into $5 \mathrm{nl}$ and injected into freshly laid single-cell-stage embryos. The animals were raised according to ${ }^{60}$. The screening of $\mathrm{F}_{0}$ animals was done by both genotyping (see above) and phenotype characterization, including pigmentation analysis and immunohistochemistry of limbs/tails, according to 39,40 . The $\mathrm{Pax}^{-1-}$ and $\mathrm{Tyr}^{-1-} \mathrm{F} 1$ animals were produced by crossing between adult $\mathrm{F}_{0}$ animals. The larvae or post-metamorphic newts were anaesthetized within $0.01 \%$ or $0.1 \%$ ethyl-p-aminobenzoate (benzocaine; Sigma) prior to imaging and tissue collection. Newts utilized for this study were processed according to Swedish Board of Agriculture animal ethical regulations (N429/12).

In situ hybridization and immunohistochemistry. The PCR fragments of Pax3 and Pax7 were cloned into TOPO cloning vectors. The digoxigenin-labeled antisense RNA probes were synthesized using T7 RNA polymerase (Roche). Wholemount in situ hybridizations were performed by using albino $\mathrm{Tyr}^{-/-} \mathrm{F} 1$ embryos according to ${ }^{62}$. In situ hybridizations on $10 \mu \mathrm{m}$ transverse sections were carried out by fixing sections in $4 \%$ formaldehyde for $15 \mathrm{~min}$, treating with $0.2 \mathrm{~N} \mathrm{HCL}$ for 12 min, washing, and then incubating in acetylation buffer $(0.1 \mathrm{M}$ trietholamine and $0.25 \%$ acetic anhydride) for $10 \mathrm{~min}$. Next, the slides were rinsed in RNase free water and permeabilized with a solution of $1 \mu \mathrm{g} \mathrm{mL}^{-1}$ Proteinase K (Roche) and 2 $\mathrm{mM} \mathrm{CaCl} 2$ for $15 \mathrm{~min}$ at $37^{\circ} \mathrm{C}$. The slides were incubated in prehybridization buffer (50\% deionized form of amide, $5 \times$ saline sodium citrate (SSC), $5 \times$ Denhardt's solution, $250 \mu \mathrm{g} \mathrm{mL}^{-1}$ yeast RNA, $500 \mu \mathrm{g} \mathrm{mL}^{-1}$ herring sperm singlestranded DNA (ssDNA); Sigma) for $2 \mathrm{~h}$ at room temperature before being incubated in hybridization solution $(50 \mathrm{ng}$ probe, $50 \%$ deionized form of amide, $5 \times$ SSC, $5 \times$ Denhardt's solution, $250 \mu \mathrm{g} \mathrm{mL}^{-1}$ yeast RNA, $500 \mu \mathrm{g} \mathrm{m}^{-1}$ herring sperm ssDNA) for $16 \mathrm{~h}$ at $60^{\circ} \mathrm{C}$. The slides were then washed with $0.2 \times$ SSC buffer containing $0.05 \%$ tween 20 at $70^{\circ} \mathrm{C}$ for $3 \times 1^{63}$. Finally, BM purple was used to visualize the signal. For immunostainings, sections were blocked with $10 \%$ donkey serum in $0.1 \%$ Triton-X for $30 \mathrm{~min}$ at room temperature. Sections were incubated with a relevant primary antibody overnight at $4 \mathrm{C}$ and with secondary antibodies for $1 \mathrm{~h}$ at room temperature. Antibodies were diluted in blocking buffer and sections were mounted in mounting medium (DakoCytomation) containing $5 \mathrm{mg} \mathrm{mL}-1$ 1 4,6-diamidino-2-phenylindole (Sigma) ${ }^{64}$.

Cryosectioning and in situ hybridization for miRNAs. Limb samples were collected at the indicated timepoints and fixed overnight at $4{ }^{\circ} \mathrm{C}$, in freshly made $4 \%$ PFA in PBS. After fixation, samples were washed three times in PBS and PBST, equilibrated in $30 \%$ sucrose in PBS, embedded in Tissue Tek (Sakura, Torrance, CA, USA) and frozen for cryosectioning. Frozen tissues were sectioned into $20 \mu \mathrm{m}-$ thick longitudinal sections.

Tissue sections were dehydrated through a $\mathrm{MeOH} / \mathrm{PBST}$ series $(25,50$, and $75 \%$ ), followed by $\mathrm{MeOH}$ for $10 \mathrm{~min}$ and rehydrated through the opposite series of $\mathrm{MeOH}$. The sections were then permeabilized with $2 \mu \mathrm{g} \mathrm{mL}^{-1}$ proteinase $\mathrm{K}$ (Thermo Scientific), inactivated with glycine solution $\left(2 \mathrm{mg} \mathrm{mL}^{-1}\right.$ in PBST) and washed in PBST. Before hybridization, the slides were incubated with hybridization buffer (50\% formamide, $5 \times \mathrm{SSC}, 50 \mu \mathrm{g} \mathrm{mL} \mathrm{m}^{-1}$ heparin, $0.2 \%$ Tween- 20 , and $100 \mu \mathrm{g}$ $\mathrm{mL}^{-1}$ yeast tRNA (Sigma)) for $1 \mathrm{~h}$ at RT. Digoxygenin-labeled locked nucleic acid (LNA) probes against the mature form of miRs-93b and 427 were custom-made and ordered from Exiqon (Denmark). The probes were diluted in hybridization buffer $(50 \mathrm{nM})$ and denatured for $5 \mathrm{~min}$ at $95^{\circ} \mathrm{C}$. Hybridization was carried overnight at $53^{\circ} \mathrm{C}$. The next day proceeded with washes in $5 \times$ SSC for $10 \mathrm{~min}$ and $0.2 \times$ SSC for $1 \mathrm{~h}$ at hybridization temperature. Then, slides were incubated in blocking buffer ( $10 \%$ sheep serum and $2 \%$ bovine serum albumin in PBST) at RT for 30 mins. Alkaline phosphatase associated anti-digoxigenin antibody (Roche) was diluted 1:1000 in blocking buffer, and slides were kept in the antibody solution at RT for $90 \mathrm{~min}$. After washing the antibody with PBST thrice, sections were incubated with freshly prepared alkaline phosphatase (AP) buffer (100 mM Tris$\mathrm{HCl}, \mathrm{pH} 9.5,50 \mathrm{mM} \mathrm{MgCl} 2,100 \mathrm{mM} \mathrm{NaCl}, 0.2 \%$ Tween-20 in water) three times for 5 min. Finally, BM Purple (Roche) was used to stain the sections, in the dark, until development of a blue/purple color. Slides were rinsed with PBST several times, and a drop of 0.5 M EDTA was added to stop the reaction. Slides were mounted with $80 \%$ glycerol and imaged using a Olympus Microscope (Center Valley, PA, USA) at $\times 10$ magnification.

Probe Sequences:

miR-93b: 5'-TCATGGAACACCTCTCAGCACTT-3

miR-427: 5'-AACCTCAACAGGAAGCACTTA-3'

scramble: 5'-GTGTAACACGTCTATACGCCCA-3'

Small RNA sequencing. Libraries from small RNAs were prepared using TruSeq Small RNA preparation kit. A total of $1 \mu \mathrm{g}$ of total RNA from 10 samples were processed according to manufacturer instructions. Libraries were sequenced on Illumina Miseq (Rapid mode), single read $1 \times 50 \mathrm{bp}$.

microRNA annotation. miRNAs were named based on the Xenopus miRNA sharing the same seed.
microRNA quantification. miRNA expression levels were quantified using miRDeep2 (quantifier.pl). To identify miRNAs associated with regeneration. Since our small-RNA dataset was intended for discovery, not differential expression analysis we did not have replicates for each source. To mitigate the lack of replicates in pursuit of miRNAs associated with regeneration, we divided the miRNA expression data into three groups: adult tissue (brain, eyes, heart, liver, lung), regenerating limb (limb $3 \mathrm{dpa}$, limb $7 \mathrm{dpa}$ ); and embryo (larvae, late embryo). Then we tested whether there was significant miRNA up or downregulation between these groups based on a Gauss-Laplace distribution, as implemented in moose ${ }^{65}$ (http:// grabherr.github.io/moose2/).

The top 10 miRNAs upregulated in the regenerating group compared to the adult group (ranked by Bonferroni-corrected $e$-value) were also the top 10 upregulated in the embryonic group compared to the adult group.

Gene expression tissue specificity. We used the tau score to quantify tissue specificity of salamander-specific orthology groups compared to orthology groups with members from other vertebrates. A tau score represents the average ratio of a gene's expression between each tissue and the tissue with the highest expression of that gene. Note that "tissue" often refers to a body part that is a composite of tissues (i.e., heart, brain, etc). A gene with equal expression across tissues will yield an average ratio of 1 . In order to make the tau score intuitive (a higher score corresponds to higher tissue specificity), the average ratio is subtracted from 1 . Therefore, a gene with equal expression across tissues will have a tau score of $1-1=0$. Conversely, if a gene is expressed in one tissue and not expressed in the others, the average ratio will approach zero and after subtracting from 1 , the tau score will approach 1 . Therefore, a gene is more tissue specific the closer its tau score is to 1.

Data availability. Datasets are deposited under BioProject PRJNA353981 and BioSample accession SAMN07571895, Runs SRR6001098-SRR6001140. Transcriptome and genome assemblies are available upon request.

Received: 6 September 2017 Accepted: 26 October 2017

Published online: 22 December 2017

\section{References}

1. Tanaka, E. M. The molecular and cellular choreography of appendage regeneration. Cell 165, 1598-1608 (2016)

2. Gemberling, M., Bailey, T. J., Hyde, D. R. \& Poss, K. D. The zebrafish as a model for complex tissue regeneration. Trends Genet. 29, 611-620 (2013).

3. Galliot, B. \& Chera, S. The Hydra model: disclosing an apoptosis-driven generator of Wnt-based regeneration. Trends Cell Biol. 20, 514-523 (2010).

4. Roberts-Galbraith, R. H. \& Newmark, P. A. On the organ trail: insights into organ regeneration in the planarian. Curr. Opin. Genet. Dev. 32, 37-46 (2015)

5. Brockes, J. P. Amphibian limb regeneration: rebuilding a complex structure. Science 276, 81-87 (1997).

6. Simon, A. \& Tanaka, E. M. Limb regeneration. Wiley interdisciplinary Rev. Dev Biol. 2, 291-300 (2013).

7. Sandoval-Guzman, T. et al. Fundamental differences in dedifferentiation and stem cell recruitment during skeletal muscle regeneration in two salamander species. Cell Stem Cell 14, 174-187 (2014).

8. Looso, M. et al. A de novo assembly of the newt transcriptome combined with proteomic validation identifies new protein families expressed during tissue regeneration. Genome Biol. 14, R16 (2013).

9. Abdullayev, I., Kirkham, M., Bjorklund, A. K., Simon, A. \& Sandberg, R. A reference transcriptome and inferred proteome for the salamander Notophthalmus viridescens. Exp. Cell. Res. 319, 1187-1197 (2013).

10. Bryant, D. M. et al. A tissue-mapped axolotl de novo transcriptome enables identification of limb regeneration factors. Cell Rep. 18, 762-776 (2017).

11. Che, R., Sun, Y., Wang, R. \& Xu, T. Transcriptomic analysis of endangered Chinese salamander: identification of immune, sex and reproduction-related genes and genetic markers. PLoS ONE 9, e87940 (2014).

12. Nakamura, K. et al. A transcriptome for the study of early processes of retinal regeneration in the adult newt, Cynops pyrrhogaster. PLoS ONE 9, e109831 (2014).

13. Hayashi, T. et al. Molecular genetic system for regenerative studies using newts. Dev. Growth Differ. 55, 229-236 (2013).

14. Lacroix, J. Etude descriptive des chromosomes en écouvillon dans le genre pleurodeles (Amphibien, Urodèle). Ann. Embryol. Morphogen. 1, 179-202 (1968).

15. Li, R. et al. ReAS: recovery of ancestral sequences for transposable elements from the unassembled reads of a whole genome shotgun. PLoS Comput. Biol. 1 , e43 (2005).

16. Bao, W., Kojima, K. K. \& Kohany, O. Repbase Update, a database of repetitive elements in eukaryotic genomes. Mobile DNA 6, 11 (2015). 
17. Grabherr, M. G. et al. Genome-wide synteny through highly sensitive sequence alignment: Satsuma. Bioinformatics 26, 1145-1151 (2010).

18. Kapitonov, V. V. \& Jurka, J. Harbinger transposons and an ancient HARBI1 gene derived from a transposase. DNA Cell Biol. 23, 311-324 (2004).

19. Sinzelle, L. et al. Transposition of a reconstructed Harbinger element in human cells and functional homology with two transposon-derived cellular genes. Proc. Natl Acad. Sci. USA 105, 4715-4720 (2008).

20. Smith, J. J., Sumiyama, K. \& Amemiya, C. T. A living fossil in the genome of a living fossil: Harbinger transposons in the coelacanth genome. Mol. Biol. Evol. 29, 985-993 (2012).

21. Ambros, V. The functions of animal microRNAs. Nature 431, 350-355 (2004).

22. Bartel, D. P. MicroRNAs: genomics, biogenesis, mechanism, and function. Cell 116, 281-297 (2004)

23. Bartel, D. P. MicroRNAs: target recognition and regulatory functions. Cell 136, 215-233 (2009)

24. Anokye-Danso, F. et al. Highly efficient miRNA-mediated reprogramming of mouse and human somatic cells to pluripotency. Cell Stem Cell 8, 376-388 (2011).

25. Tang, G. Q. \& Maxwell, E. S. Xenopus microRNA genes are predominantly located within introns and are differentially expressed in adult frog tissues via post-transcriptional regulation. Genome Res. 18, 104-112 (2008).

26. Watanabe, T. et al. Stage-specific expression of microRNAs during Xenopus development. FEBS Lett. 579, 318-324 (2005).

27. Giraldez, A. J. et al. MicroRNAs regulate brain morphogenesis in zebrafish. Science 308, 833-838 (2005)

28. Suh, M. R. et al. Human embryonic stem cells express a unique set of microRNAs. Dev. Biol. 270, 488-498 (2004).

29. Greve, T. S., Judson, R. L. \& Blelloch, R. microRNA control of mouse and human pluripotent stem cell behavior. Annu. Rev. Cell. Dev. Biol. 29, 213-239 (2013).

30. Chen, P. Y. et al. The developmental miRNA profiles of zebrafish as determined by small RNA cloning. Genes Dev. 19, 1288-1293 (2005).

31. Shubin, N. H., Daeschler, E. B. \& Coates, M. I. The early evolution of the tetrapod humerus. Science 304, 90-93 (2004).

32. Frobisch, N. B. \& Shubin, N. H. Salamander limb development: integrating genes, morphology, and fossils. Dev. Dyn. 240, 1087-1099 (2011).

33. Friedli, M. \& Trono, D. The developmental control of transposable elements and the evolution of higher species. Annu. Rev. Cell Dev. Biol. 31, 429-451 (2015).

34. Friedli, M. et al. Loss of transcriptional control over endogenous retroelements during reprogramming to pluripotency. Genome Res. 24, 1251-1259 (2014).

35. Yanai, I. et al. Genome-wide midrange transcription profiles reveal expression level relationships in human tissue specification. Bioinformatics 21, 650-659 (2005).

36. Baddar, N. W., Woodcock, M. R., Khatri, S., Kump, D. K. \& Voss, S. R. Sal-Site: research resources for the Mexican axolotl. Methods Mol. Biol. 1290, 321-336 (2015).

37. Keinath, M. C. et al. Initial characterization of the large genome of the salamander Ambystoma mexicanum using shotgun and laser capture chromosome sequencing. Sci. Rep. 5, 16413 (2015).

38. Knapp, D. et al. Comparative transcriptional profiling of the axolotl limb identifies a tripartite regeneration-specific gene program. PLOS ONE 8, e61352 (2013).

39. Epstein, J. A. Pax3 and vertebrate development. Methods Mol. Biol. 137, $459-470$ (2000)

40. Joven, A., Morona, R., Gonzalez, A. \& Moreno, N. Spatiotemporal patterns of Pax3, Pax6, and Pax7 expression in the developing brain of a urodele amphibian, Pleurodeles waltl. J. Comp. Neurol. 521, 3913-3953 (2013).

41. Buckingham, M. \& Relaix, F. PAX3 and PAX7 as upstream regulators of myogenesis. Semin. Cell Dev. Biol. 44, 115-125 (2015).

42. Sun, C. et al. LTR retrotransposons contribute to genomic gigantism in plethodontid salamanders. Genome Biol. Evol. 4, 168-183 (2012).

43. Morgan, G. T. Lampbrush chromosomes and associated bodies: new insights into principles of nuclear structure and function. Chromosome Res. 10, 177-200 (2002).

44. Dunis, D. A. \& Namenwirth, M. The role of grafted skin in the regeneration of x-irradiated axolotl limbs. Dev. Biol. 56, 97-109 (1977).

45. Kuang, S., Chargé, S. B., Seale, P., Huh, M. \& Rudnicki, M. A. Distinct roles for Pax7 and Pax3 in adult regenerative myogenesis. J. Cell Biol. 172, 103-113 (2006).

46. Wang, H. \& Simon, A. Skeletal muscle dedifferentiation during salamander limb regeneration. Curr. Opin. Genet. Dev. 40, 108-112 (2016).

47. Giraldez, A. J. et al. Zebrafish MiR-430 promotes deadenylation and clearance of maternal mRNAs. Science 312, 75-79 (2006).

48. Lund, E., Liu, M., Hartley, R. S., Sheets, M. D. \& Dahlberg, J. E. Deadenylation of maternal mRNAs mediated by miR-427 in Xenopus laevis embryos. RNA. 15, 2351-2363 (2009).
49. Jaylet, A. [Change in the karyotype by a homozygous pericentric inversion in the newt Pleurodeles waltlii Michahelles]. Chromosoma 35, 288-299 (1971).

50. Gallien, C. L. [The karyotype of the urodele Pleurodeles poireti Gervais Comparative study of karyotypes in the genus Pleurodeles].C. R. Acad. Sci. Hebd. Seances Acad. Sci. D 262, 122-125 (1966).

51. Penrad-Mobayed, M., Kanhoush, R. \& Perrin, C. Tips and tricks for preparing lampbrush chromosome spreads from Xenopus tropicalis oocytes. Methods 51, 37-44 (2010).

52. Dolezel, J., Greilhuber, J. \& Suda, J. Estimation of nuclear DNA content in plants using flow cytometry. Nat. Protoc. 2, 2233-2244 (2007).

53. Licht, L. E. \& Lowcock, L. A. Genome size and metabolic-rate in salamanders. Comp. Biochem. Phys. B 100, 83-92 (1991).

54. Morescalchi, A. \& Olmo, E. Sirenids - family of polyploid urodeles. Experientia 30, 491-492 (1974)

55. Lizana, M. et al. Determination of cellular DNA content of Iberian salamanders by flow cytometry. Amphibia-Reptilia 21, 411-418 (2000).

56. Felsenstein, J. Mathematics vs. evolution: mathematical evolutionary theory. Science 246, 941-942 (1989).

57. Edgar, R. C. MUSCLE: multiple sequence alignment with high accuracy and high throughput. Nucleic Acids Res. 32, 1792-1797 (2004).

58. Anisimova, M. \& Gascuel, O. Approximate likelihood-ratio test for branches: a fast, accurate, and powerful alternative. Syst. Biol. 55, 539-552 (2006).

59. Guindon, S. \& Gascuel, O. A simple, fast, and accurate algorithm to estimate large phylogenies by maximum likelihood. Syst. Biol. 52, 696-704 (2003).

60. Joven, A., Kirkham, M. \& Simon, A. Husbandry of Spanish ribbed newts (Pleurodeles waltl). Methods Mol. Biol. 1290, 47-70 (2015).

61. Hayashi, T. \& Takeuchi, T. Gene manipulation for regenerative studies using the Iberian ribbed newt, Pleurodeles waltl. Methods Mol. Biol. 1290, 297-305 (2015).

62. Monsoro-Burq, A. H. A rapid protocol for whole-mount in situ hybridization on Xenopus embryos. CSH Prot. 2007, pdbprot4809, https://doi.org/10.1101/ pdb.prot4809 (2007).

63. Kirkham, M., Hameed, L. S., Berg, D. A., Wang, H. \& Simon, A. Progenitor cell dynamics in the Newt Telencephalon during homeostasis and neuronal regeneration. Stem cell reports 2, 507-519 (2014).

64. Wang, H. et al. Turning terminally differentiated skeletal muscle cells into regenerative progenitors. Nat. Commun. 6, 7916 (2015)

65. Berghoff, B. A., Karlsson, T., Källman, T., Wagner, E. G. H. \& Grabherr, M. G. RNA-sequence data normalization through in silico prediction of reference genes: the bacterial response to DNA damage as case study. BioData Min. 10 30 (2017).

66. Kumar, S., Stecher, G., Suleski, M. \& Hedges, S. B. TimeTree: a resource for timelines, timetrees, and divergence times. Mol. Biol. Evol. 34, 1812-1819 (2017).

67. Miyake, A. \& Araki, M. Retinal stem/progenitor cells in the ciliary marginal zone complete retinal regeneration: a study of retinal regeneration in a novel animal model. Dev. Neurobiol. 74, 739-756 (2014).

68. Yoshii, C., Ueda, Y., Okamoto, M. \& Araki, M. Neural retinal regeneration in the anuran amphibian Xenopus laevis post-metamorphosis: transdifferentiation of retinal pigmented epithelium regenerates the neural retina. Dev. Biol. 303, $45-56$ (2007).

69. Lozito, T. P. \& Tuan, R. S. Lizard tail skeletal regeneration combines aspects of fracture healing and blastema-based regeneration. Development 143, 2946-2957 (2016).

70. Berg, D. A. et al. Efficient regeneration by activation of neurogenesis in homeostatically quiescent regions of the adult vertebrate brain. Development 137, 4127-4134 (2010)

71. Roco, Á. et al. Coexistence of $\mathrm{Y}, \mathrm{W}$, and $\mathrm{Z}$ sex chromosomes in Xenopus tropicalis. Proc. Natl Acad. Sci. USA 112, E4752-E4761 (2015).

72. Session, A. M. et al. Genome evolution in the allotetraploid frog Xenopus laevis. Nature 538, 336-343 (2016).

73. Sessions, S. K. Evolutionary cytogenetics in salamanders. Chromosome Res. 16, 183-201 (2008)

74. Liew, W. C. \& Orbán, L. Zebrafish sex: a complicated affair. Brief Funct. Genomics 13, 172-187 (2014).

\section{Acknowledgements}

We thank Elly Tanaka and colleagues for sharing the axolotl genome assembly prior to publication. We acknowledge continuous support from members of the Frisén Lab (KI/ $\mathrm{CMB}$ ), Science for Life Laboratory, the National Genomics Infrastructure (NGI) the Swedish National Infrastructure for Computing (SNIC) and Uppsala Multidisciplinary Center for Advanced Computational Science (UPPMAX) for providing assistance in massive parallel sequencing and computational infrastructure (projects b2015079, b2015188, b2016417, SNIC 2016/1-20 and SNIC 2016/1-251). We thank Agustin Gonzalez and his lab (UCM, Madrid) for facilitating the embryos from which our initial colony was established. Mihaela Martis (BILS - SciLifeLab) is acknowledged for assistance concerning transcriptome assembly, Sarantis Giatrellis (CMB - KI) for assistance measuring nuclear DNA content and Karen Echeverri for facilitating miRNA in situ 
hybridization experiments. We thank Marc Friedlander for advice regarding miRNA prediction. A.E. is supported by NIH Ruth Kirschstein postdoctoral fellowship F32GM117806. This work was supported by grants from the National Natural Science Foundation of China (31771617) to H.W., the European Research Council, the Swedish Research Council, Wenner-Gren Foundation and Cancerfonden to A.S

\section{Author contributions}

A.E. conceived the study, assembled and annotated the P. walt transcriptome, predicted miRNAs in the genome and transcriptome, merged the N. viridescens transcriptomes, performed orthology group analysis and wrote the manuscript. H.W. performed the Pax3 and Pax7 in situ hybridization, gene editing, genotype and phenotype analysis and wrote the corresponding part of the manuscript. C.T.-L. assembled the P. waltl genome, annotated its protein-coding genes and edited the manuscript. A.J. established the $P$. waltl colony and breeding program, helped in the design of the sampling strategy, performed karyotyping from primary cell cultures, CRISPR/Cas9 egg injections, phenotype characterization, breeding, and assisted with figure preparation for the manuscript. M.P.-M. prepared, acquired and analyzed lampbrush chromosome images. Z.Y. preformed nuclear DNA quantification. Y.A .assisted with gene expression analysis. N.Z. performed parts of the transposable element analysis. G.B. performed miRNA in situ hybridization. A.K. and L.S.H. performed in situ hybridization. I.A. and

R.S. contributed to $P$. walt transcriptome assembly. M.G. designed the transposable element analysis, assembled repeat libraries, and identified repeat content, transposable element distribution and wrote the manuscript. B.A. participated in the planning of the study and supervised the genome sequencing and analysis and edited the manuscript. A. S. conceived the study, supervised all aspects of the project and wrote the manuscript.

\section{Additional information}

Supplementary Information accompanies this paper at doi:10.1038/s41467-017-01964-9.

Competing interests: The authors declare no competing financial interests.

Reprints and permission information is available online at http://npg.nature.com/ reprintsandpermissions/

Publisher's note: Springer Nature remains neutral with regard to jurisdictional claims in published maps and institutional affiliations.

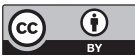

Open Access This article is licensed under a Creative Commons Attribution 4.0 International License, which permits use, sharing, adaptation, distribution and reproduction in any medium or format, as long as you give appropriate credit to the original author(s) and the source, provide a link to the Creative Commons license, and indicate if changes were made. The images or other third party material in this article are included in the article's Creative Commons license, unless indicated otherwise in a credit line to the material. If material is not included in the article's Creative Commons license and your intended use is not permitted by statutory regulation or exceeds the permitted use, you will need to obtain permission directly from the copyright holder. To view a copy of this license, visit http://creativecommons.org/ licenses/by/4.0/.

(C) The Author(s) 2017 